\title{
NON-INVASIVE OCULAR RIGIDITY MEASUREMENT: A DIFFERENTIAL TONOMETRY APPROACH
}

\author{
Efstathios T. Detorakis ${ }^{1,2}$, Emmanuela Tsaglioti ${ }^{1}$, George Kymionis ${ }^{1,2}$
}

Institute of Vision \& Optics, University of Crete, Greece ${ }^{1}$; Department of Ophthalmology, University Hospital of Heraklion, Greece $^{2}$

Summary: Purpose: Taking into account the fact that Goldmann applanation tonometry (GAT) geometrically deforms the corneal apex and displaces volume from the anterior segment whereas Dynamic Contour Tonometry (DCT) does not, we aimed at developing an algorithm for the calculation of ocular rigidity (OR) based on the differences in pressure and volume between deformed and non-deformed status according to the general Friedenwald principle of differential tonometry. Methods: To avoid deviations of GAT IOP from true IOP in eyes with corneas different from the "calibration cornea" we applied the previously described Orssengo-Pye algorithm to calculate an error coefficient "C/B". To test the feasibility of the proposed model, we calculated the OR coefficient (r) in 17 cataract surgery candidates ( 9 males and 8 females). Results: The calculated $\mathrm{r}$ according to our model (mean $\pm \mathrm{SD}$, range) was $0.0174 \pm 0.010(0.0123-0.022) \mathrm{mmHg} / \mu \mathrm{L}$. A negative statistically significant correlation between axial length and $\mathrm{r}$ was detected whereas correlations between $\mathrm{r}$ and other biometric parameters examined were statistically not significant. Conclusions: The proposed method may prove a valid non-invasive tool for the measurement method of OR, which could help in introducing OR in the decision-making of the routine clinical practice.

Key words: Cornea; Sclera; Ultrasonography

\section{Introduction}

Ocular Rigidity (OR) is an important property of the ocular tissues associated with their resistance to mechanical deformation (1). From a purely mathematical standpoint, OR refers to the correlation between pressure and volume in a chamber filled with incompressible content (1-4). This mathematical correlation is affected by the elastic properties of the chamber walls (1-4). However, in the case of the eyeball, which is also filled with incompressible content (aqueous humor and gel-like vitreous body), rigidity is affected by not only the elastic properties of the sclera and cornea but also by other factors, such as the vascular uveal layer (5). The latter displays a dynamic change in its elastic properties due to constant changes in the amount of blood contained in uveal vessels in response to a variety of physiological factors, such as the cardiac cycle, respiratory movements or intraocular pressure (IOP) changes (6). Moreover, the internal compartmental architecture of the eyeball, organized as anterior segment (filled by dynamically flowing queous humor) and posterior segment (filled by the more static vitreous body gel), complicate its bio-mechanical behaviour, which has accordingly been described as poro-elastic, rather than elastic (7). In the case of the cornea, bio-mechanical behaviour also includes a visco-elastic or anisotropic element, implying that that the rate at which a load is applied changes the measured value for cornea's Young's modulus (7-9). The latter describes the resistance of corneal tissue to mechanical deformation and corresponds to the relation between tensile strain and tensile stress of corneal tissue (9). Reported corneal Young's modulus values range from $0.159 \mathrm{MPa}$ to $57 \mathrm{Mpa}$ (mean $0.29 \pm 0.06 \mathrm{Mpa}$ ) (7-9), reflecting the complexity of ex-vivo corneal bio-mechanical behaviour.

Several attempts have so far been made to measure OR (1, 10-13). The initial land-mark studies of Friedenwald in 1937, who employed a differential tonometry methodology (using indentation or indentation and applanation tonometry) in human cadaver eyes, have resulted in a purpose-designed chart providing an OR coefficient (on the average $0.0215 \mathrm{mmHg} / \mu \mathrm{L})(1)$. According to the Friedenwald model, the rigidity coefficient $(\mathrm{K})$ may be calculated as:

$$
K=\frac{\log I O P_{1}-\log I O P_{2}}{V_{1}-V_{2}}
$$

Where $\mathrm{P}_{1}$ and $\mathrm{P}_{2}$ as well as $\mathrm{V}_{1}$ and $\mathrm{V}_{2}$ refer to respective values of intraocular pressure and volume (1). However, this approach has received criticism because the conditions in living human eyes are notably different from those in cadaveric eyes due to blood circulation and the lack of 
post-mortem connective tissue changes (11). Other researchers have since then attempted to measure OR using a variety of methodologies (10-13). More recently, Pallikaris et al. have reported accurate rigidity measurements by inserting a manometric catheter into the anterior chamber and directly measuring pressure-volume changes (11). However, this approach is invasive (requires a surgical intervention) and thus cannot be used in the every-day clinical practice. In fact, an important obstacle in including OR in the routine clinical decision-making has been the lack of a simple, accurate and, more importantly, non-invasive methodology for its quantitative assessment, despite the fact that OR may be involved in a variety of clinical situations, such as glaucoma, age-related macular degeneration (AMD) or presbyopia (3). Based on this point, we aimed at developing such a methodology by mathematically analyzing differential tonometry readings between applanation and non-applanation tonometers in association with other clinical parameters, all easily recordable in a non-invasive manner.

\section{Material and Methods}

This study was conducted at the Department of Ophthalmology of the University Hospital of Heraklion, in Crete, Greece and the protocol was approved by the local ethical committee. Cataract candidate patients were included in the study. A mathematical algorithmic tool to measure OR based on Friedenwald's principle but using ophthalmic parameters recorded in a non-invasive manner was developed. The tool was examined in a group of cataract surgery candidates. Eyes with a history of trauma, surgical procedures or inflammatory conditions as well as eyes with glaucoma and a history of anti-glaucomatous eye drop use were excluded. Moreover, eyes with corneal dystrophies or other ocular surface conditions, such as pterygium, or posterior segment abnormalities, such as staphylomas, were also excluded. None of the eyes included had astigmatism over 3.00D and the spherial equivalent was below $8.00 \mathrm{D}$ in all cases. Overall, 17 patients ( 9 males and 8 females) were included in the study.

\section{Theoretical concept for the formation of the algorithmic tool}

Taking into account that the basic definition of OR relates with the association between pressure and volume changes in the eyeball, we explored the possibility to take advantage of the "delta", i.e. the difference $(\Delta \mathrm{IOP})$ between applanation (GAT) and non-applanation (DCT) tonometry, in association with the volume displaced during the applanation phase of GAT. The latter depends on a modified "Imbert-Fick" concept, according to which the pressure (P) within a sphere with ideally elastic and thin walls equals to the force necessary to applanate a part of the sphere (W) divided by the area applanated (A), whereas the force necessary to distort the cornea (B) and surface tension (S) are also involved: $W+S=P \times A+B$ (14). In the case of DCT, the Pascal principle applies, referring to the equality of forces created by actual IOP, capillary traction and ocular rigidity on the anterior corneal surface (2). The basic difference between the 2 methods refers to the lack of applanation of the anterior corneal surface, thus lack of induced corneal deformation and respective volume displacement in the case of DCT (2). During GAT the displaced volume corresponds to an ellipsoid cup (Figure 1). By applying the general ellipsoid equation (15) of

$$
\frac{x^{2}}{a^{2}}+\frac{y^{2}}{b^{2}}+\frac{z^{2}}{c^{2}}=1
$$

in which $\mathrm{a}, \mathrm{b}$ and $\mathrm{c}$ refer to the width along the $\mathrm{x}-, \mathrm{y}$ - and $\mathrm{z}$-axes, respectively, whereas $\mathrm{X}(0,0, \mathrm{x})$ is a point on the ellipsoid surface such as $-\mathrm{c} \times \mathrm{c}$, the ellipsoid cup volume can been calculated by a standard calculating machine (16) as:

$$
V=\pi a b\left(\frac{2 c}{3}-x+\frac{x^{3}}{3 c^{2}}\right)
$$

To apply the general Friedenwald principle of differential pressure and volume we assumed that the initial eye volume corresponds with the corneal status during DCT (i.e. non-deformed) whereas the final ocular volume corresponds with the corneal status during GAT (i.e. with corneal deformation and associated volume displacement). Moreover, taking into account the lack of deformation in DCT, we assumed that DCT IOP reading approximates true IOP without the need of further corrections. However, according to the previously published model of Orssengo-Pye (17), GAT IOP readings equal true IOP when corneal parameters are in agreement with the geometrical characteristics of the so-called "calibrated cornea", such as CCT of $520 \mu \mathrm{m}$ and mean external radius of curvature of $7.8 \mathrm{~mm}$. To correct for deviations of GAT IOP from true IOP in eyes with corneas different from the "calibration cornea" we applied the Orssengo-Pye algorithm (17):

$$
I O P_{P}=\frac{I O P_{G}}{C / B}
$$

In the error coefficient " $\mathrm{C} / \mathrm{B}$ " $\mathrm{B}$ corresponds to the $\mathrm{IOP}_{\mathrm{G}}$ of the calibrated cornea and $\mathrm{C}$ corresponds to the IOP of the measured cornea (17). To calculate $\mathrm{B}$ and $\mathrm{C}$ the following equations were applied (17):

$$
\begin{gathered}
B=\frac{0.6 \cdot \pi \cdot R \cdot\left(R-\frac{t}{2}\right) \cdot \sqrt{1-v^{2}}}{t^{2}} \\
C=\frac{\pi \cdot R \cdot\left(R-\frac{t}{2}\right)^{2} \cdot(1-v)}{A \cdot t}
\end{gathered}
$$


Where $\mathrm{R}$ corresponds to the anterior corneal curvature, $\mathrm{t}$ corresponds to the central corneal thickness (CCT), $v$ is the Poisson's index for the cornea (0.49) and A is the area of applanation. Instead of a CCT of $520 \mu \mathrm{m}$ as originally proposed by Orssengo-Pye we used the mean CCT of patients included in this study $(549 \mu \mathrm{m})$. In accordance with the initial differential tonometry equation described by Friedenwald but incorporating the error coefficient $\mathrm{C} / \mathrm{B}$ for the deviation of the measured cornea for the calibrated cornea in GAT IOP, as well as the mean corneal Young's modulus (E) previously reported (9), and thus measured " $r$ " using the following algorithm:

$$
\mathrm{r}=\left[\left(I O P_{\text {Pascal }}-I O P_{\text {Goldmann }} / \Delta V\right) \times C / B\right] \times E
$$

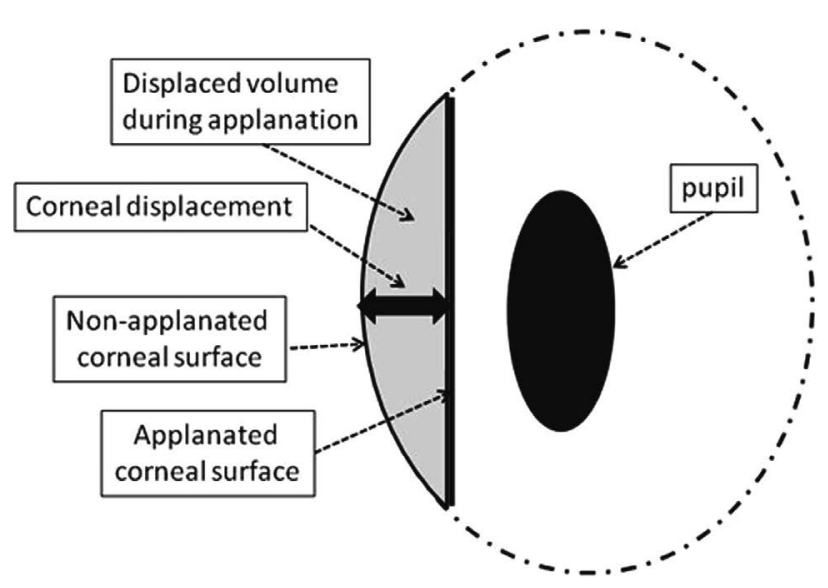

Fig. 1: Applanated and non-applanated corneal surfaces in GAT with respective ellipsoid cup volume displacement.

\section{Clinical application of the proposed algorithm}

All patients underwent a typical preoperative clinical examination, including IOP measurements by both Goldmann Applanation Tonometry (GAT) and Dynamic Contour Tonometry (DCT), ultrasonic axial length (AL) and anterior corneal surface curvature measurements, ultrasonic central corneal thickness (CCT) measurement as well as measurements of the maximal eyeball diameter (corresponding to the equatorial region) along both transverse (TD) and coronal (CD) planes. DCT (SMT Swiss Microtechnology AG, Port, Switzerland) was performed immediately after instillation of proparacaine eye drops in the examined eyes (3 readings Q1-Q3, as per manufacturer instructions were taken and the mean value recorded). GAT was then performed (after the application of a fluorescein strip at the lower conjunctival fornix). Five GAT measurements were taken in eaqch eye and the average was recored as the GAT IOP. CCT, AL, TD and CD were measured with the Alcon OcuScan ${ }^{\circledR} \mathrm{RxP}$ Ophthalmic Ultrasound System (Alcon laboratories, Alcon, Irvine, CA, USA), employing a $20 \mathrm{Mhz}$ probe for $\mathrm{CCT}$,

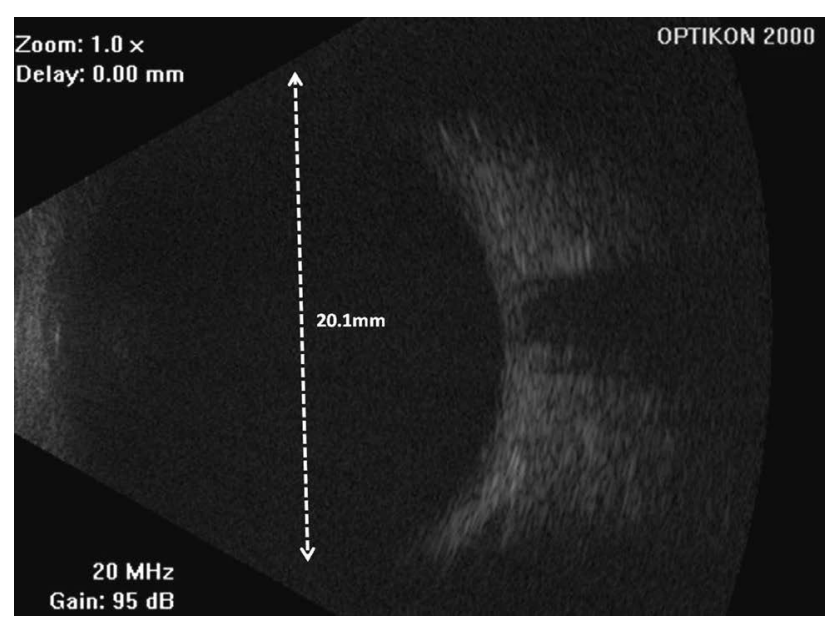

Fig. 2: Ophthalmic B-scan image with measurement of maximal (equatorial) globe diameter (in this case $20.1 \mathrm{~mm}$, shown with dashed lines), along the coronal plane.

(with a resolution of $\pm 1 \mu \mathrm{m}$ and an accuracy of $\pm 5 \mu \mathrm{m}$ ) and a $10 \mathrm{Mhz}$ probe (with a resolution of $\pm 0.1 \mathrm{~mm}$ and a theoretical accuracy of $\pm 0.05 \mathrm{~mm}$ ) for AL, TD and CD. TD and $\mathrm{CD}$ were recorded using a B-scan mode of ultrasonic imaging by obtaining a cross-sectional image of the eye at the transverse (nasal-temporal diameter) and coronal (superior-inferior diameter) planes, respectively. Measurements were taken with the built-in measurement tool by placing the measurement cursors along the largest diameter of the examined eye, on the transverse and coronal planes (Figure 2). For all ultrasonic parameters, which were performed by the same experienced examiner (ET), 10 successive measurements were taken and the mean was recorded.

\section{Statistical analysis}

The examination correlations between the parameters recorded was performed with Pearson's bivariate correlation coefficient. Statistical significance was set at 0.05 . Statistical analyses were performed with the statistical package SPSS 8.0 (SPSS, Chicago, IL, USA).

\section{Results}

Measurements (mean $\pm \mathrm{SD}$, range) of the recorded parameters in the group of patients studied is presented in Table 1. The application of the algorithm mentioned in the case series included in this feasibility study rendered an index (r) of corneal rigidity of $0.0174 \pm 0.010(0.0123-0.022) \mathrm{mmHg} / \mu \mathrm{L}$. The delta recorded was $1.78 \pm 0.71(-0.10-2.08) \mathrm{mmHg}$. The correlation between delta and $r$ was statistically significant (Pearson's bivariate correlation coefficient 0.803, $\mathrm{p} \approx 0.00$ ) (Figure 3). A negative correlation between $\mathrm{AL}$ and $\mathrm{r}$ was detected, although at a borderline statistical signficance level (Pearson's bivariate correlation coefficient $-0.482, p=0.048$ ) (Figure 4). On the contrary, the corre- 
Tab. 1: Parameters recorded with mean, SD and range values.

\begin{tabular}{|l|c|c|c|}
\hline \multicolumn{1}{|c|}{ Parameter } & Mean & SD & Range \\
\hline AL (mm) & 23.63 & 1.16 & $21.19-25.84$ \\
\hline R (mm) & 7.82 & 0.29 & $7.44-8.48$ \\
\hline CCT $(\boldsymbol{\mu m})$ & 548.94 & 37.80 & $462-594$ \\
\hline Goldmann (mmHg) & 16.29 & 3.46 & $10-20$ \\
\hline Pascal (mmHg) & 16.25 & 3.48 & $11.4-22.8$ \\
\hline $\begin{array}{l}\text { Nasal-Temporal } \\
\text { diameter (mm) }\end{array}$ & 18.95 & 0.98 & $17.56-20.60$ \\
\hline $\begin{array}{l}\text { Superior-Inferior } \\
\text { diameter (mm) }\end{array}$ & 18.81 & 0.94 & $17.33-20.45$ \\
\hline Ocular Rigidity & 0.0173 & 0.0070 & $0.0080-0.0329$ \\
\hline DIOP (mmHg) & 1.78 & 0.71 & $0.8-3.3$ \\
\hline
\end{tabular}

lation between $r$ and the the vertical or horizontal maximal diamterers of the eyeball was statistically not significant (Pearson's bivariate correlation coefficient). Moreover, the correlations between CCT, corneal curvature, DCT or GAT was statistically not significant (Pearson's bivariate correlation coefficient).

\section{Discussion}

This study examined the feasibility of using "delta" as a metric for the calculation of corneal rigidity, based on the mathematical analysis of various biometric indices. Results imply that "delta" may be used to calculate a coefficient for

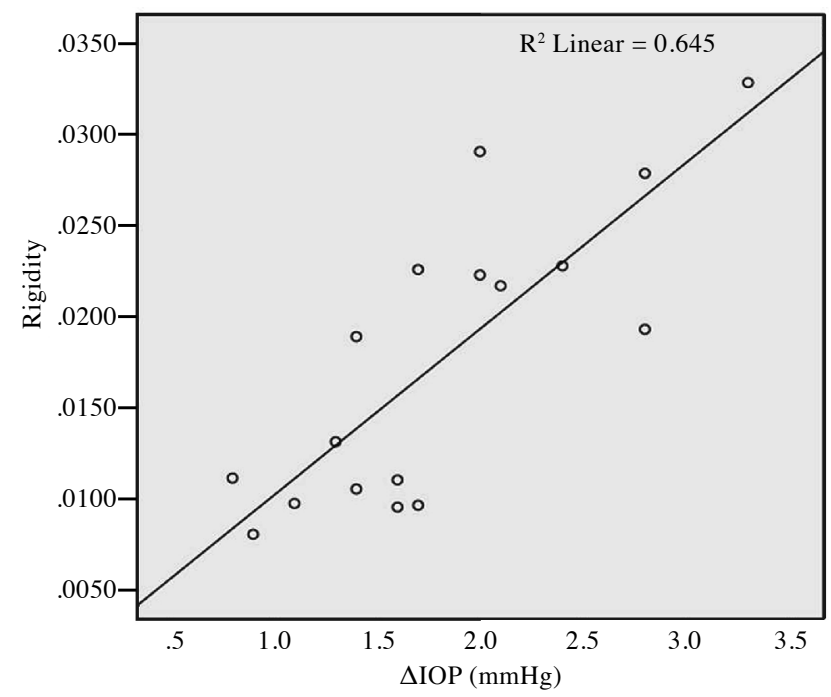

Fig. 3: Correlation between $\Delta \mathrm{IOP}$ and Rigidity (r) in the group of patients studied.
OR (r) in a non-invasive manner applicable in the every-day clinical practice.

The accurate non-invasive measurement of OR has been the target of various research projects so far $(1,3$, $7,18)$. Calculating " $r$ " for a particular eye is very important since " $r$ " varies considerably between eyes and this variation may have clinical implications for the course of several conditions, such as glaucoma (12), presbyopia (3) or AMD (19). Although in the case of glaucoma there have been reports for the assessment of ocular biomechanical properties through the use of modalities such as the Ocular Response Analyzer (ORA), so far results are inconclusive (20). In the case of ORA, the association between corneal hysteresis and the OR or Young's modulus of the cornea is unclear and hysteresis has been shown to decrease during aging, when the cornea is known to stiffen, as well as to decrease after the cornea has been stiffened by cross-linking techniques $(3,20)$. Nevertheless, the accurate assessment of OR may also be of value in the case of pseudoexfoliation syndrome and exfoliation glaucoma, in which the biomechanical behaviour of affected tissues may be changed though the accumulation of pseudoexfoliative material per se or through alterations in blood supply (21-23). In a previously published paper by Liu \& Roberts, a model of simulation of corneal biomechanical behaviour was proposed and it was shown that variations in corneal biomechanics, expressed by differences in corneal Young's modulus, may actually affect IOP to a greater extent than corneal thickness or curvature (24). Furthermore, the use of corneal cross-linking in the management of keratoconus and other corneal conditions such as post-LASIK ectasia may also have implications for OR due to significant changes in corneal biomechanical properties (25) and the introduction of a non-invasive quantitative method to assess OR as the

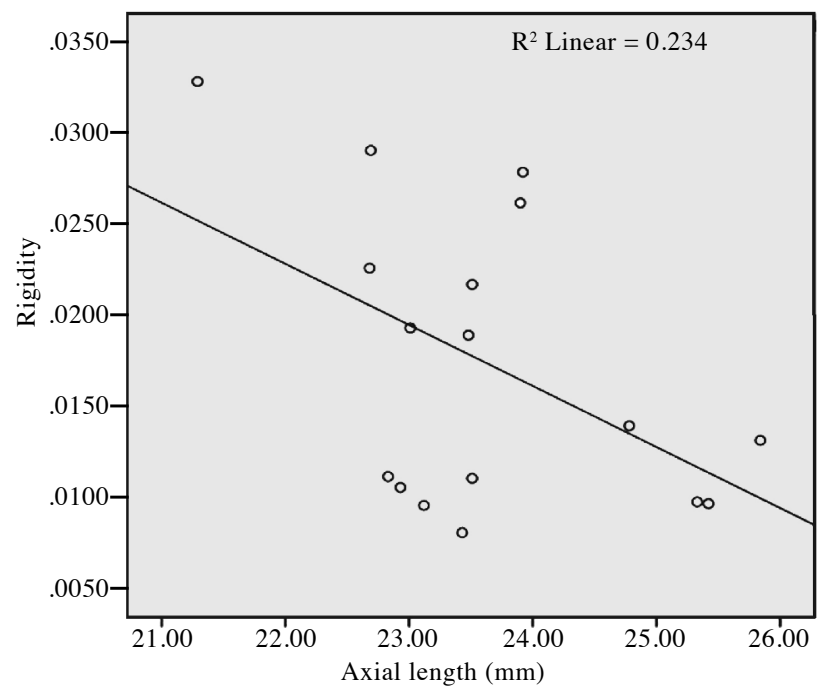

Fig. 4: Correlation between AL and Rigidity (r) in the group of patients studied. 
one described in the present study, could provide important clinical information.

Moreover, it has become evident that the elastic properties of the ocular walls, especially those of the cornea, affect significantly the accuracy of tonometric methods, such as the indentation (SchiÖtz) tonometry $(2,14)$. Although GAT was initially considered to be immune from such effects, it soon became evident that it is was significantly affected by a variety of corneal parameters, including CCT (26), corneal curvature (27), corneal astigmatism (28), AL (29) or even the bio-mechanical properties of corneal collagen, most of them related with OR (2). DCT may be less affected by CCT, compared with GAT, however it may in fact be more affected by corneal curvature, since during DCT the concave surface of the tonometer head (which has a pre-determined radius of curvature corresponding to the average corneal curvature) has to conform geometrically with the anterior corneal surface, which varies in curvature between different eyes (2). Nevertheless, the fundamental difference between GAT and DCT lies in the event of applanation in GAT and respective lack of applanation in DCT (2). The average volume displaced during applanation has been reported to be $0.5 \mu \mathrm{L}$, but it differs between different eyes, depending on corneal geometry (the volumetric displacement in applanation tonometry of a cornea with a radius of curvature of $4.5 \mathrm{~mm}$ has been reported to be $0.995 \mathrm{ml}$, while that of a cornea with a radius of $15.5 \mathrm{~mm}$ has been reported to be $0.281 \mathrm{ml})(3,30)$. The methodology proposed in this study takes advantage of this volume, which is calculated in a customized fashion for the individual eye examined, based on a three-dimensional ellipsoid model of the eyeball. A modified concept of differential tonometry, based on the originally proposed model by Friedenwald for SchiÖtz tonometry performed with 2 different weights (or for SchiÖtz and GAT tonometries), is then applied between GAT and DCT.

The average " $r$ " calculated for the eyes included in this case series is very close to previously reported " $r$ " scores, calculated with other methodologies in previous studies, such as those by Friedenwald (0.0215) (1), Goldmann (0.020) (13), Drance (0.0217) (10), Agarwal (0.0217) (12) and Pallikaris (0.0126) (11). Moreover, a negative association between AL and $\mathrm{r}$ has also been previously reported (4). These consistencies enhance the validity of the proposed methodology. On the other hand, weaknesses of the present study are the small number of patients included, the requirement for the availability of a non-applanation tonometer and the lack of a purpose-designed independent validation method for the calculation of " $r$ " applied on the same case series. Accordingly, a further step in the examination of the validity of the methodology proposed could be the comparison of results with results from a different method of OR measurement performed on the same series of eyes. Moreover, assumptions were also employed in the approach described in this study, such as the fact that DCT IOP corresponds with the "true" IOP in the non-deformed corneal status whereas results may better describe corneal rigidity, rather than ocular rigidity, since deformation corresponds to corneal geometry. It could also be argued that since the volume of the anterior chamber is around $250 \mu \mathrm{l}$, and the volume of the anterior chamber displaced by Goldmann tonometry is around $0.5 \mu \mathrm{l}(3)$, i.e. $0.2 \%$ of the total volume, it may be hard to predict ocular rigidity changes based on the volume displaced by Goldmann tonometry alone. However, previously published models for the calculation of OR are also based on very small changes in ocular geometry (e.g. a reduction in AL of 14.2-23 $\mu \mathrm{m})(18)$, implying that the mathematical tools employed may have the power to assess OR based on such small deviations.

The obvious advantage of the present methodology is its non-invasive nature, which enables its application in the every-day clinical practice. A previous study attempted to measure OR by examining changes in AL (associated with ocular volume changes) caused by the oral administration of acetazolamide $(500 \mathrm{mg})$, which would later result in a respective IOP reduction (18). Although this methodology may also be considered non-invasive, it requires, the systemic administration of acetazolamide (which may be contra-indicated in some patients) and may be more time-consuming than the present approach, which could render its application difficult in a busy clinical setting. The methodology presented in this study overcomes these obstacles and, if proved valid, may be a useful clinical tool in the customized assessment of OR, enabling its active involvement in the decision making for a variety of clinical situations.

\section{References}

1. Friedenwald JS. Tonometer calibration; an attempt to remove discrepancies found in the 1954 calibration scale for Schiotz tonometers. Trans Am Acad Ophthalmol Otolaryngol 1957; 61: 108-222.

2. Detorakis ET, Arvanitaki V, Pallikaris IG, Kymionis G, Tsilimbaris MK. Applanation Tonometry versus Dynamic Contour Tonometry in Eyes Treated with Latanoprost. J Glaucoma 2010; 19: 194-198.

3. Detorakis ET, Pallikaris IG. Ocular rigidity: biomechanical role, in vivo measurements and clinical significance. Clin Experiment Ophthalmol 2013; 41: 73-81. doi: 10.1111/j.1442-9071.2012.02809.x. Epub 2012 Jul 2. Review.

4. Dastiridou AI, Ginis H, Tsilimbaris M, et al. Ocular rigidity, ocular pulse amplitude, and pulsatile ocular blood flow: the effect of axial length. Invest Ophthalmol Vis Sci 2013 Mar 1; 54: 2087-92.

5. Friberg TR, Lace JW. A comparison of the elastic properties of human choroid and sclera. Exp Eye Res 1988; 47: 429-436.

6. Hommer A, Fuchsjäger-Mayrl G, Resch H, Vass C, Garhofer G, Schmetterer L. Estimation of ocular rigidity based on measurement of pulse amplitude using pneumotonometry and fundus pulse using laser interferometry in glaucoma. Invest Ophthalmol Vis Sci 2008; 49: 4046-50.

7. Detorakis ET, Drakonaki EE, Tsilimbaris MK, Pallikaris IG, Giarmenitis S. Real-time ultrasound elastographic imaging of ocular and periocular tissues: a feasibility study. Ophthalmic Surg Lasers Imaging 2010; 41: 135-41.

8. Detorakis ET, Drakonaki EE, Ginis H, Karyotakis N, Pallikaris IG. Evaluation of iridociliary and lenticular elasticity using shear-wave elastography in rabbit eyes. Acta Medica (Hradec Králové) 2014; 57: 9-14. 
9. Hamilton KE, Pye DC. Young's modulus in normal corneas and the effect on applanation tonometry. Optom Vis Sci 2008; 85: 445-50.

10. Drance SM. The coefficient of scleral rigidity in normal and glaucomatous eyes. Arch Ophthalmol 1960; 63: 668-74.

11. Pallikaris IG, Kymionis GD, Ginis HS, Kounis GA, Tsilimbaris MK. Ocular rigidity in living human eyes. Invest Ophthalmol Vis Sci 2005; 46: 409-14.

12. Agrawal KK, Sharma DP, Bhargava G, Sanadhya DK. Scleral rigidity in glaucoma, before and during topical antiglaucoma drug therapy. Indian J Ophthalmol 1991; 39: $85-86$.

13. Goldmann H, Schmidt T. Friedenwald's rigidity coefficient. Ophthalmologica 1957; 133: 330-335.

14. Goldmann $\mathrm{H}$. Un nouveau tonomètre à aplanation [A new applanation tonometer] Bull Mem Soc Fr Ophtalmol 1954; 67: 474-7.

15. Harris WF. Curvature of ellipsoids and other surfaces. Ophthalmic Physiol Opt 2006; 26: 497-501.

16. http://www.codecogs.com/library/maths/geometry/volume/ellipsoidal_cap.php

17. Orssengo GJ, Pye DC. Determination of the true intraocular pressure and modulus of elasticity of the human cornea in vivo. Bull Math Biol 1999; 61: 551-72.

18. Ebneter A, Wagels B, Zinkernagel MS. Non-invasive biometric assessment of ocular rigidity in glaucoma patients and controls. Eye 2009; 23: 606-611.

19. Pallikaris IG, Kymionis GD, Ginis HS, Kounis GA, Christodoulakis E, Tsilimbaris MK. Ocular rigidity in patients with age-related macular degeneration. Am J Ophthalmol 2006; 141: 611-615.

20. Shah S, Laiquzzaman M, Bhojwani R, Mantry S, Cunliffe I. Assessment of the biomechanical properties of the cornea with the ocular response analyzer in normal and keratoconic eyes. Invest Ophthalmol Vis Sci 2007; 48: 3026-3031.
21. Detorakis ET, Chrysochoou F, Paliobei V, et al. Evaluation of the acoustic function in pseudoexfoliation syndrome and exfoliation glaucoma: audiometric and tympanometric findings. Eur J Ophthalmo. 2008; 18: 71-6.

22. Detorakis ET, Koukoula S, Chrisohoou F, Konstas AG, Kozobolis VP. Central corneal mechanical sensitivity in pseudoexfoliation syndrome. Cornea 2005; 24 : 688-91.

23. Detorakis ET, Achtaropoulos AK, Drakonaki EE, Kozobolis VP. Hemodynamic evaluation of the posterior ciliary circulation in exfoliation syndrome andexfoliation glaucoma. Graefes Arch Clin Exp Ophthalmol 2007; 245: 516-21.

24. Liu J, Roberts CJ. Influence of corneal biomechanical properties on intraocular pressure measurement: quantitative analysis. J Cataract Refract Surg 2005; 31 : 146-55.

25. Sorkin N, Varssano D. Corneal collagen crosslinking: a systematic review. Ophthalmologica 2014; $232: 10-27$.

26. Ehlers N, Bramsen T, Sperling S. Applanation tonometry and central corneal thickness Acta Ophthalmol (Copenh) 1975; 53: 34-43.

27. Mark H. Corneal curvature in applanation tonometry Am J Ophthalmol 1973; 76 : 223-224.

28. Francis BA, Hsieh A, Lai MY, et al. Los Angeles Latino Eye Study Group. Effects of corneal thickness, corneal curvature, and intraocular pressure level on Goldmann applanation tonometry and dynamic contour tonometry. Ophthalmology 2007; 114: 20-26.

29. Mark H, Robbins KP, Mark TL. Axial length in applanation tonometry. J Cataract Refract Surg 2002; 28: 504-506.

30. Abdalla MI, Hamdi M. Applanation ocular tension in myopia and emmetropia. Br J Ophthalmol 1970; 54: 122-125.

Received: $17 / 05 / 2015$

Accepted in revised form: 22/08/2015

\section{Corresponding author:}

Efstathios T. Detorakis, MD, PhD, FEBO, Department of Ophthalmology, University Hospital of Heraklion, 71110, Heraklion, Crete, Greece; e-mail: detorakis@hotmail.com 